\title{
Poroid Hymenochaetaceae (Basidiomycota) from Parque Estadual da Serra do Mar, Núcleo Santa Virgínia, São Paulo, Brazil
}

\author{
Ricardo Matheus Pires ${ }^{1,2}$ \& Adriana de Mello Gugliotta ${ }^{1}$
}

\begin{abstract}
This survey presents the first poroid Hymenochaetaceae checklist from Parque Estadual da Serra do Mar, São Paulo state, Brazil, where 24 species are presented. Fulvifomes melleoporus, Fuscoporia chrysea, Fuscoporia rhabarbarina, Inonotus linteus, Inonotus portoricensis, Phellinus grenadensis, Phellinus roseocinereus and Phellinus undulatus are cited for São Paulo state for the first time, and Fulvifomes glancescens is a new record to Brazil. Full description of the new record for Brazil, comments on the new records for São Paulo state, photos of the new records, as well as an identification key for the 24 confirmed species found in the park are presented.
\end{abstract}

Key words: Atlantic Forest, diversity, fungi, polypores.

\section{Resumo}

Esta pesquisa apresenta a primeira lista de espécies de fungos poroides da família Hymenochaetaceae do Parque Estadual da Serra do Mar, São Paulo, Brasil, onde 24 espécies são registradas pela primeira vez na localidade. Fulvifomes melleoporus, Fuscoporia chrysea, Fuscoporia rhabarbarina, Inonotus linteus, Inonotus portoricensis, Phellinus grenadensis, Phellinus roseocinereus e Phellinus undulatus são citados para o estado de São Paulo pela primeira vez e Fulvifomes glaucescens é um novo registro para o Brasil. São apresentadas descrição do registro novo para o Brasil, comentários sobre os registros novos para o estado de São Paulo e fotos dos novos registros, além de uma chave de identificação para as 24 espécies encontradas no parque. Palavras-chave: Mata Atlântica, diversidade, fungos, políporos.

\section{Introduction}

The family Hymenochaetaceae Donk (1948) is included into the order Hymenochaetales and 27 genera are accepted in the family, with 487 species (Kirk et al. 2008). All the species of this group live as saprophytes or parasites on a wide diversity of angiosperms and gymnosperms playing a fundamental role in wood rotting and being part of the carbon cycling in the forest ecosystems; however, they are understudied in most tropical regions (Ryvarden 1991).

This group is defined by simple septa in the generative hyphae, xanthochroic reaction, yellow to deep brown basidiomata, frequent occurrence of setae, and they cause white rot (Ryvarden 1991; Dai 2010). However, recent molecular studies have shown that many genera into the family are polyphyletic and new genera are being created to include the different species, once traditional characters used before to separate the genera occur in parallel in different groups (Wagner \& Fischer 2001; Wagner \& Fischer 2002; Dai et al. 2008; He \& Dai 2012; Vlasák et al. 2013).

Brazilian Atlantic forest is one of the most important tropical forests in the world, considered a hotspot of diversity, the flora and fauna may include $1-8 \%$ of the world's total species (da Silva $\&$ Casteleti 2003). Ribeiro et al. (2009) estimates a remaining coverage in a range of $11.4 \%$ to $16 \%$, which demonstrates the extremely degraded state of the Atlantic forest, warning to urgent conservation and restoration actions.

\footnotetext{
'Instituto de Botânica de São Paulo, Núcleo de Pesquisa em Micologia, Av. Miguel Stefano 3687, Água Funda, 04301-012, São Paulo, SP, Brasil.

${ }^{2}$ Author for correspondence: sals.bio@gmail.com
} 
According to Baltazar \& Gibertoni (2009), the family Hymenochaetaceae in the Brazilian Atlantic forest is represented for 103 species. The genus with the highest number of recorded species is Phellinus Quél. (42 species), followed by Hymenochaete Lév. (27 species). There are several studies on the diversity of fungi in the Brazilian Atlantic forest (Góes-Neto et al. 2000; Groposo \& Loguercio-Leite 2005; Drechsler-Santos et al. 2008; Westphalen et al. 2010; Motato-Vásquez \& Gugliotta 2013; Westphalen \& Silveira 2013; Motato-Vásquez et al. 2014) but this study presents the first poroid Hymenochaetaceae checklist for Parque Estadual da Serra do Mar, the largest Atlantic forest reserve in the São Paulo State. In this study, full description of the new record for Brazil, comments on the new records to São Paulo State, photos of the new records, as well as an identification key for the 24 confirmed species found in the park are presented.

\section{Material and Methods}

Parque Estadual da Serra do Mar (PESM) occupies 315,000 ha of São Paulo state and Núcleo Santa Virgínia is one of its eight nuclei. Situated in the municipalities of São Luiz do Paraitinga and Natividade da Serra, the Núcleo Santa Virgínia covers a total area of 17,000 ha and is located in the geographical coordinates $45^{\circ} 03^{\prime} \mathrm{W}$ and $23^{\circ} 24^{\prime} \mathrm{S}$ to $45^{\circ} 11^{\prime} \mathrm{W}$ and $23^{\circ} 17^{\prime} \mathrm{S}$. The altitudinal range varies from $860 \mathrm{~m}$ to $1650 \mathrm{~m}$, maximum temperatures $35^{\circ} \mathrm{C}$, average of $21^{\circ} \mathrm{C}$ and a low of $-3^{\circ} \mathrm{C}$, its vegetation is characterized by Mountain Dense Ombrophilous Forest (Evergreen Forest from Plateau), with some small Altitude Fields sections (Steppe) and Fog Forest (Forest of Serra do Mar Crest) (Joly et al. 2012).
Bimonthly, samples were collected from April 2013 to April 2014. The sampling followed the recommendations of Fidalgo \& Bononi (1984). Macro and microscopic characters of the basidiomata were analyzed to identify the specimens, following the recommendations of Fidalgo (1968), Ryvarden (1991) and Teixeira $(1962,1995)$. Melzer's reagent was used to check amyloid and dextrinoid reactions in micro structures. The basidiospores dimensions were obtained as Coelho (2005) proposed, by calculating the ratio $\mathrm{Q}=\mathrm{L} / \mathrm{W}$ and average of $\mathrm{Q}_{\mathrm{m}}$ (where $\mathrm{L}=$ length and $\mathrm{W}=$ width).

The main identification keys were Larsen \& Cobb-Poulle (1990), Gilbertson \& Ryvarden (1986), Gilbertson \& Ryvarden (1987), Ryvarden (2004) and Groposo et al. (2007). All materials collected are deposited in the Herbarium SP.

\section{Results and Discussion}

Hymenochaetaceae is represented in the Parque Estadual da Serra do Mar by 24 species, all new records to the park, and nine represent new records for São Paulo State, Fulvifomes melleoporus (Murrill) Baltazar \& Gibertoni, Fuscoporia chrysea (Lév.) Baltazar \& Gibertoni, Fuscoporia rhabarbarina (Berk.) Groposo, Log.Leite \& Góes-Neto, Inonotus linteus (Berk. \& M.A. Curtis) Teixeira, Inonotus portoricensis (Overh.) Baltazar \& Gibertoni, Phellinus grenadensis (Murrill) Ryvarden, Phellinus roseocinereus (Murrill) D.A. Reid and Phellinus undulatus (Murrill) Ryvarden. Also, Fulvifomes glaucescens (Petch) Y.C. Dai is recorded for the first time in Brazil.

\section{Key to species of poroid Hymenochaetaceae from Parque Estadual da Serra do Mar}

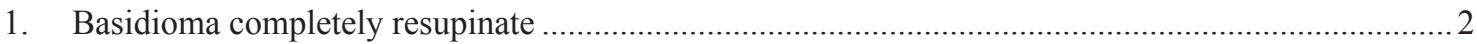

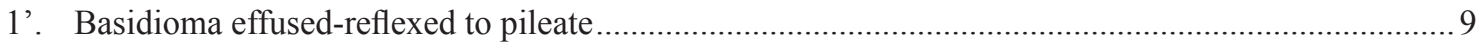

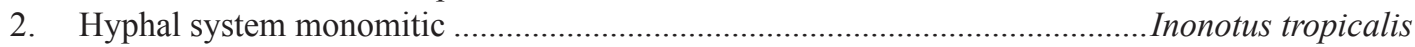

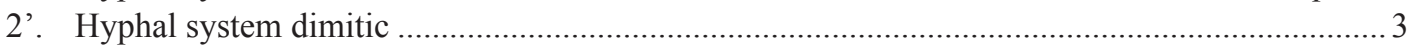

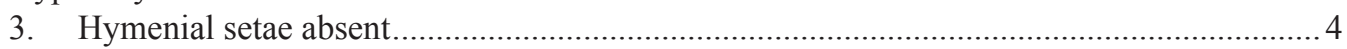

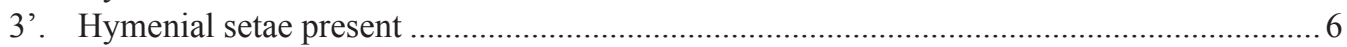

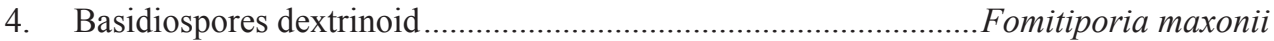

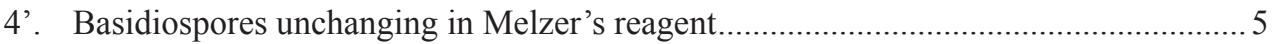

5. Pores 4-6 per mm, basidiospores pale golden yellow

Fulvifomes melleoporus

5'. Pores 6-8 per mm, basidiospores rusty brown............... Fomitiporella umbrinella 6. Basidiospores dextrinoid ........................................Fomitiporia bambusarum

6'. Basidiospores unchanging in Melzer's reagent............................................ 7 
7. Hymenial setae hooked to straight Phellinus undulatus

7'. Hymenial setae only straight. .8

8. Basidiospores cylindrical

Fuscoporia ferrea

8'. Basidiospores broadly ellipsoid to subglobose

Fulvifomes glaucescens

9. Hyphal system monomitic 10

9'. Hyphal system dimitic, or at least dimitic in the trama. 12

10. Setal hyphae present Inonotus portoricensis

10'. Setal hyphae absent. . .11

11. Pores $4-6$ per $\mathrm{mm}$ Cyclomyces iodinus

11'. Pores 7-9 per mm Cyclomyces tabacinus

12. Hymenial setae absent . .13

12'. Hymenial setae present 17

13. Basidiospores dextrinoid Fomitiporia apiahyna

13'. Basidiospores unchanging in Melzer's reagent

14. Basidiospores 3.0-3.5 × $3.0 \mu \mathrm{m}$.

14'. Basidiospores longer.

15. Pores $7-9$ per $\mathrm{mm}$ 14

15 . Pores up to 7 per $\mathrm{mm}$ nata 16. Basidiospores $4.0-6.0 \times 3.0-5.0 \mu \mathrm{m}$, pores $4-7$ per $\mathrm{mm}$.. Phellinus grenadensis

16'. Basidiospores 5.5-7.0 × 4.0-5.0 $\mu \mathrm{m}$, pores (3) $4-5$ per mm Fulvifomes rimosus

17. Hymenial setae hooked to straight

7'. Hymenial setae only straight

Fuscoporia wahlbergii

18. Pileus with distinct black crust

Fuscoporia rhabarbarina

18'. Pileus without a black crust 19

19. Pores 5-7 per mm 20

19'. Pores 7-11 per mm 22

20. Basidiospores $2.0-2.5 \mu \mathrm{m}$ wide

Fuscoporia chrysea

20'. Basidiospores wider .21

21. Basidiospores ellipsoid to ovoid, hyaline, $4.0-5.0 \times 3.0-3.5 \mu \mathrm{m}$..

Fuscoporia gilva

21'. Basidiospores ellipsoid to subglobose, yellowish, 4.8-5.5 $\times 3.9-4.6 \mu \mathrm{m}$.... Inonotus linteus

22. Pore surface cinnamon to deep umber brown ... Fuscoporia callimorpha

22'. Pore surface yellow-fulvous, yellow to reddish brown ...

23. Basidiospores broadly oval, $3.5-4.0 \times 2.0-3.0$ $\mu \mathrm{m}$...............Phellinus roseocinereus

23'. Basidiospores broadly ellipsoid, 4.5-6.0 × 3.5-5.0 $\mu \mathrm{m}$ ...... Fuscoporia senex 
Species list

Cyclomyces iodinus (Mont.) Pat., Essai taxonomique sur les familles et les genres des Hyménomycètes 98. 1900.

Description: Ryvarden (2004).

Materials examined: São Luiz do Paraitinga, Parque Estadual da Serra do Mar, Núcleo Santa Virgínia, Trilha do Corcovado, 11.VI.2013, R.M. Pires 49 (SP), R.M. Pires 53 (SP); Trilha do Poço do Pito, 7.VIII.2013, R.M. Pires et al.112 (SP); Trilha do Garcês, 12.II.2014, R.M. Pires et al.244 (SP).

Cyclomyces tabacinus (Mont.) Pat., Essai Tax. Hyménomyc. (Lons-le-Saunier): 98. 1900.

Description: Ryvarden (2004).

Material examined: São Luiz do Paraitinga, Parque Estadual da Serra do Mar, Núcleo Santa Virgínia, Trilha do Ipiranga, 17 IV 2013, R.M. Pires et al.5 (SP).

Fomitiporella umbrinella (Bres.) Murrill, North American Flora 9(1): 13. 1907.

Description: Ryvarden \& Johansen (1980) and Núñez \& Ryvarden (2000).

Material examined: São Luiz do Paraitinga, Parque Estadual da Serra do Mar, Núcleo Santa Virgínia, Trilha do Pau de Bala, 25.IV.2014, R.M. Pires 276 (SP).

Fomitiporia apiahyna (Speg.) Robledo, Decock \& Rajchenberg s.l., Mycologia 102(6): 1315. 2010.

Description: Ryvarden (2004).

Material examined: São Luiz do Paraitinga, Parque Estadual da Serra do Mar, Núcleo Santa Virgínia, Trilha do Garcês, 12.II.2014, R.M. Pires et al.253 (SP).

Fomitiporia bambusarum (Rick) Campos-Santana \& Decock, Cryptogamie, Mycologie 36(1): 48. 2015. Description: Larsen \& Cobb-Poulle (1990).

Material examined: São Luiz do Paraitinga, Parque Estadual da Serra do Mar, Núcleo Santa Virgínia, Trilha do Corcovado, 11.VI.2013, R.M. Pires et al.50 (SP).

Fomitiporia maxonii Murrill, North American Flora 9(1): 11. 1907.

Description: Ryvarden (2004).

Material examined: São Luiz do Paraitinga, Parque Estadual da Serra do Mar, Núcleo Santa Virgínia, Trilha do Pirapitinga, 27.IV.2014, R.M. Pires et al.327 (SP).

Fulvifomes glaucescens (Petch) Y.C. Dai, Fungal Diversity 45: 192. 2010.

Figs. 1d-g; 3a

Material examined: São Luiz do Paraitinga, Parque Estadual da Serra do Mar, Núcleo Santa Virgínia, Trilha do Poço do Pito, 26.IV.2014, R.M. Pires et al.307 (SP). Additional material examined: SRI LANKA. NUWARA ELIYA: Hakgala, T. Petch (?), V.1913, K (M) 163705, Type.
Basidioma: annual, resupinate, becoming woody hard upon drying, up to $20 \mathrm{~cm}$ long, $10 \mathrm{~cm}$ wide and $0.2 \mathrm{~cm}$ thick, dark brown to black with age. Pore surface: greyish brown when fresh, dark brown when bruised, sometimes with a glaucous shade; cracked when dry, margin rust-brown, very narrow to almost lacking, pores circular to angular, 7-8(9) per $\mathrm{mm}$. Hyphal system: dimitic, generative hyphae with simple septa, hyaline to pale yellow in $\mathrm{KOH}$, simple to slightly branched, up to $4 \mu \mathrm{m}$ wide; skeletal hyphae yellowish to pale rusty brown, thick-walled with a narrow lumen, unbranched or with rare branched, 2-5 $\mu \mathrm{m}$ in diam. Sterile elements: hymenial setae frequent, ventricose to subulate, straight, $14-24 \times 4.5-7.0 \mu \mathrm{m}$. Basidia: not seen. Basidiospores: broadly ellipsoid to subglobose, yellowish, thick-walled, smooth, IKI-, 3.9-4.6 $\times 3.1-3.7 \mu \mathrm{m}, \mathrm{Q}=1.2-1.4, \mathrm{Q}_{\mathrm{m}}=1.2$.

Remarks: this species is known from Africa and Asia (Ryvarden \& Johansen 1980; Larsen \&

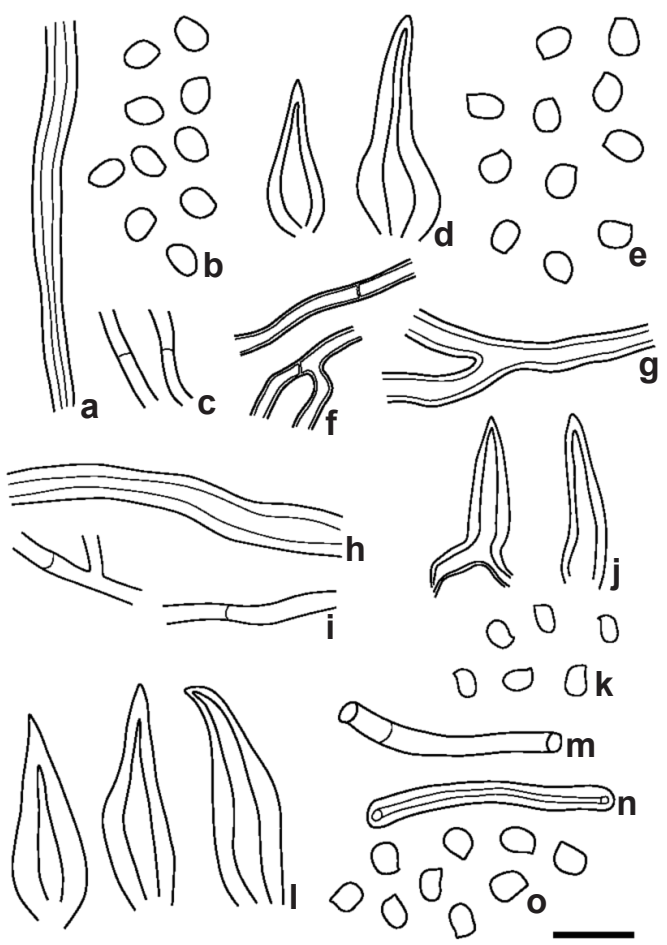

Figure 1-a-c. Fulvifomes melleoporus - a. skeletal hyphae; b. basidiospores; c. generative hyphae. d-g. Fulvifomes glaucescens - d. hymenial setae; e. basidiospores; $\mathrm{f}$. generative hyphae; g. skeletal hyphae. h-k. Fuscoporia rhabarbarina - h. skeletal hyphae; i. generative hyphae; j. hymenial setae; k. basidiospores. 1-o. Fuscoporia chrysea - 1. hymenial setae; m. generative hyphae; n. skeletal hyphae; o. basidiospores. Scale bar $=10 \mu \mathrm{m}$. 
Cobb-Poulle 1990). The first record from America was in México (Valenzuela et al. 2012) and now is recorded for Brazil. The tiny pores in the resupinate basidiomata and the yellowish, thick-walled and medium size basidiospores can define this species. Nuñes \& Ryvarden (2000) concern this is a somewhat confusing species and the size of the setae is a variable characteristic. In Dai (2010) reviewing for Chinese Hymenochaetaceae, he brought up some disagreements between authors concerning the validity of this species.

Fulvifomes melleoporus (Murrill) Baltazar \& Gibertoni, Mycotaxon 111: 205. 2010.

Figs. 1a-c; $3 \mathrm{~b}$

Description: Larsen \& Cobb-Poulle (1990) and Ryvarden (2004).

Material examined: São Luiz do Paraitinga, Parque Estadual da Serra do Mar, Núcleo Santa Virgínia, Trilha do Pau de Bala, 25.IV.2014, R.M. Pires et al.280 (SP).

Remarks: F. melleoporus has perennial and resupinate basidiomata, with a golden brown to dark purplish brown pore surface. The lack of setae and small pale golden yellow basidiospores (4.0$4.6 \times 3.2-3.7 \mu \mathrm{m})$ are characteristic for recognizing this species. It is known in three Brazilian States: Mato Grosso do Sul, Bahia and Rio Grande do Norte (Maia et al. 2015), and now in São Paulo.
Fulvifomes nilgheriensis (Mont.) Bondartseva \& S. Herrera, Mikologiya i Fitopatologiya 26(1): 13. 1992. Description: Larsen \& Cobb-Poulle (1990) and Ryvarden (2004).

Material examined: São Luiz do Paraitinga, Parque Estadual da Serra do Mar, Núcleo Santa Virgínia, Trilha do Poço do Pito, 7.VIII.2013, R.M. Pires et al. 115 (SP).

Fulvifomes rimosus (Berk.) Fiasson \& Niemelä, Karstenia 24(1): 26. 1984.

Description: Ryvarden (2004).

Material examined: São Luiz do Paraitinga, Parque Estadual da Serra do Mar, Núcleo Santa Virgínia, Trilha do Corcovado, 11.VI.2013, R.M. Pires et al. 68 (SP).

Fuscoporia callimorpha (Lév.) Groposo, Log.Leite \& Góes-Neto, Mycotaxon 101: 57. 2007.

Description: Ryvarden \& Johansen (1980).

Material examined: São Luiz do Paraitinga, Parque Estadual da Serra do Mar, Núcleo Santa Virgínia, Trilha do Pirapitinga (na sub-trilha do olho d'água), 29.X.2013, R.M. Pires et al. 135 (SP).

Fuscoporia chrysea (Lév.) Baltazar \& Gibertoni, Mycotaxon 111: 206. 2010. Figs. 11-o; 3c Description: Ryvarden \& Johansen (1980) and Ryvarden (2004).

Materials examined: São Luiz do Paraitinga, Parque Estadual da Serra do Mar, Núcleo Santa Virgínia, Trilha

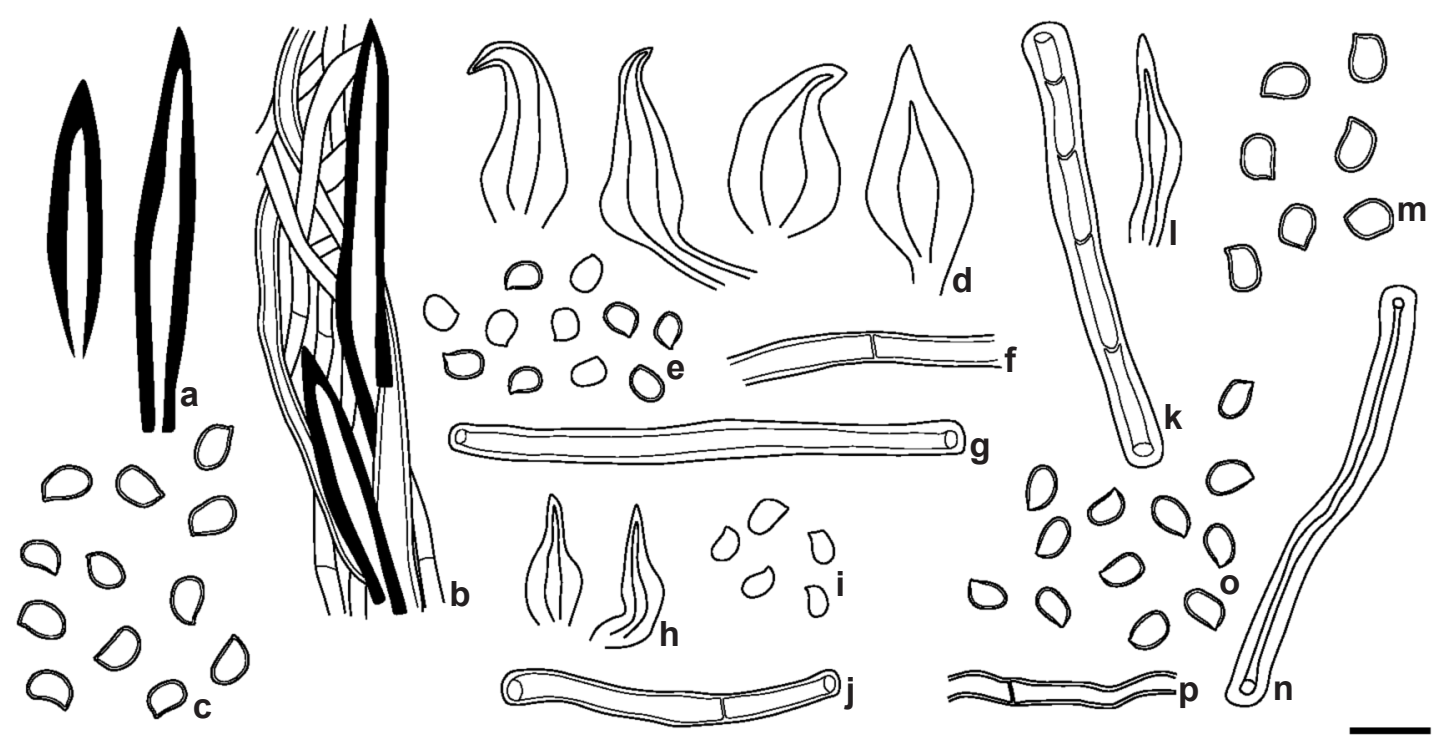

Figure 2 - a-c. Inonotus portoricensis - a. setal hyphae; b. trama; c. basidiospores. d-g. Phellinus undulatus - d. hymenial setae; e. basidiospores; f. generative hyphae; g. skeletal hyphae. h-j. Phellinus roseocinereus - h. hymenial setae; i. basidiospores; j. generative hyphae. k-m. Inonotus linteus - k. generative hyphae; l. hymenial setae; m. basidiospores. n-p. Phellinus grenadensis - n. skeletal hyphae; o. basidiospores; $\mathrm{p}$. generative hyphae. Scale bar $(\mathrm{a}-\mathrm{b})=20 \mu \mathrm{m} ;(\mathrm{c}-\mathrm{p})=10 \mu \mathrm{m}$. 
do Pirapitinga, 13.VI.2013, R.M. Pires et al. 83 (SP); 27.IV.2014, R.M. Pires et al.330 (SP).

Additional material examined: PERNAMBUCO: São Vicente Férrer, Mata do Estado (Sirijí), J.M. Baltazar et al., 1164 (URM).

Remarks: This species is characterized macroscopically by its effused reflexed basidioma, with small and imbricate pilei and microscopically by the common hymenial setae $(24-30 \times 6.0-8.0$ $\mu \mathrm{m})$ and basidiospores broadly ellipsoid and hyaline $(3.5-4.1 \times 2.0-2.5 \mu \mathrm{m})$. The concept of this species is not well established and Ryvarden (2004) suggests that the original description is only an early stage of development of basidioma, and
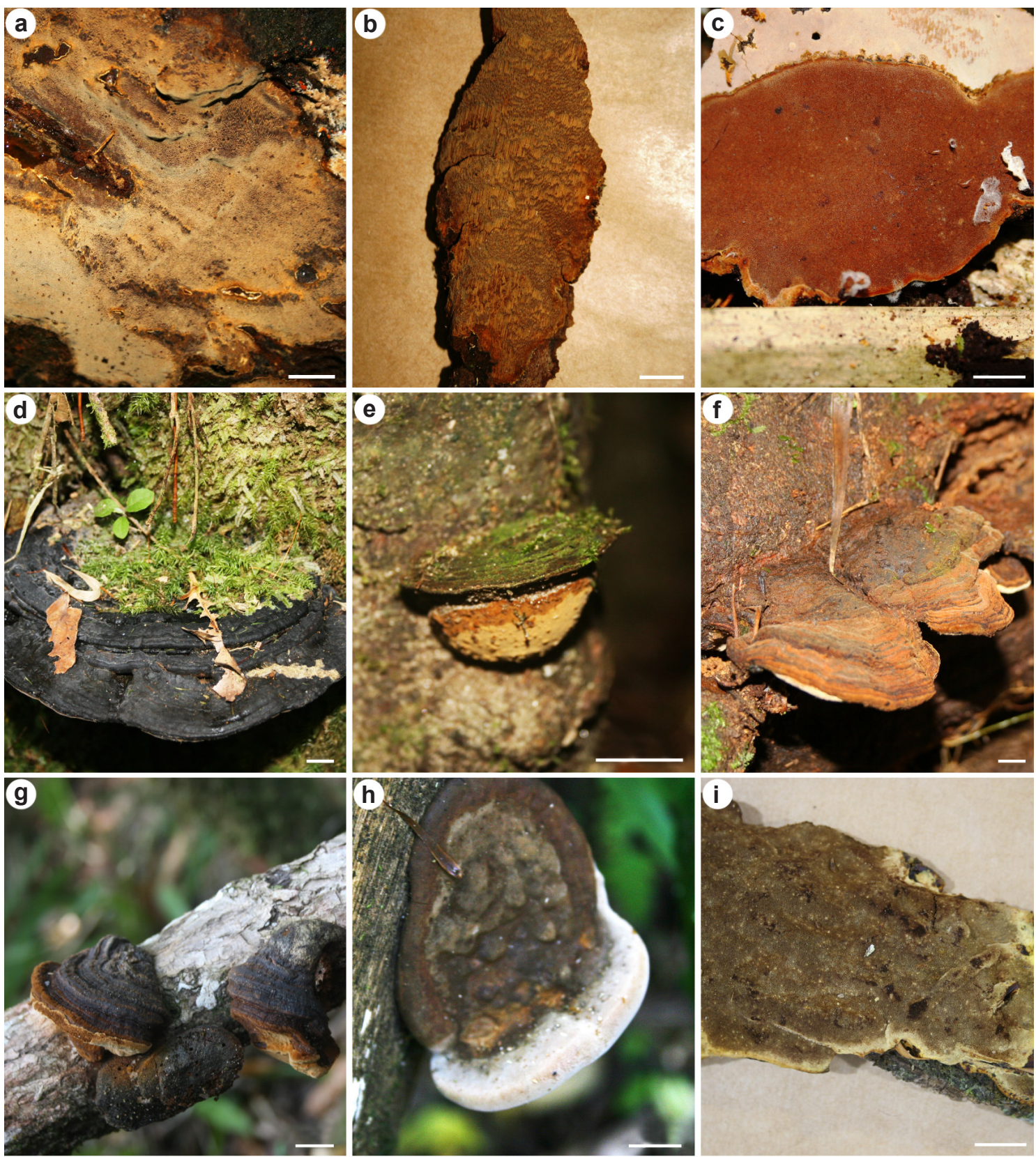

Figure 3 - a. Fulvifomes glaucescens; b. Fulvifomes melleoporus; c. Fuscoporia chrysea; d. Fuscoporia rhabarbarina; e. Inonotus linteus; f. Inonotus portoricensis; g. Phellinus grenadensis; h. Phellinus roseocinereus; i. Phellinus undulatus. Scale bar $=1 \mathrm{~cm}$. 
a bigger pileus could be formed in later stages. Fuscoporia gilva is quite similar, differing by surface color and pore size (brown and 6-8 per $\mathrm{mm}$ in $F$. gilva and yellow and 5-6 per $\mathrm{mm}$ in $F$. chrysea). This collection represents the first record to São Paulo State and to Southern Brasil. So far, this species only have been recorded in Pernambuco, Northeast Brazil (Maia et al. 2015).

Fuscoporia ferrea (Pers.) G. Cunn., Bulletin of the New Zealand Department of Industrial Research 73: 7. 1948.

Description: Ryvarden \& Gilbertson (1994).

Material examined: São Luiz do Paraitinga, Parque Estadual da Serra do Mar, Núcleo Santa Virgínia, Trilha do Pirapitinga, 18.XII.2013, R.M. Pires et al. 194 (SP).

Fuscoporia gilva (Schwein.) T. Wagner \& M. Fisch., Mycologia 94(6): 1013. 2002.

Description: Ryvarden (2004).

Materials examined: São Luiz do Paraitinga, Parque Estadual da Serra do Mar, Núcleo Santa Virgínia, Trilha do Ipiranga, 17.IV.2013, R.M. Pires et al. 17 (SP), R.M. Pires et al. 21 (SP); Trilha do Corcovado, 11.VI.2013, R.M. Pires et al. 54 (SP); Trilha do Poço do Pito, 7.VIII.2013, R.M. Pires et al. 104 (SP), R.M. Pires et al. 108 (SP); 30.X.2013, R.M. Pires et al. 149 (SP), R.M. Pires et al. 151 (SP); Trilha do Pirapitinga, 31.X.2013, R.M. Pires et al. 168 (SP); 10.II.2014, R.M. Pires et al. 220 (SP); 27.IV.2014, R.M. Pires et al. 323 (SP).

Fuscoporia rhabarbarina (Berk.) Groposo, Log.Leite \& Góes-Neto, Mycotaxon 101: 61. 2007. Figs. 1h-k; 3d

Description: Ryvarden \& Johansen (1980) and Gerber \& Loguercio-Leite (1997).

Material examined: São Luiz do Paraitinga, Parque Estadual da Serra do Mar, Núcleo Santa Virgínia, Trilha do Poço do Pito, 7.VIII.2013, R.M. Pires et al. 100 (SP).

Remarks: F. rhabarbarina is a pileate, wood and perenial species, with hyphal system dimitic and hymenial setae $20-30 \times 4.0-5.0 \mu \mathrm{m}$. It is characterized by the glabrous black pilei surface and small basidiospores ellipsoid and hyaline $(3.5-4.0 \times 2.0-3.0 \mu \mathrm{m})$. Known in Rio Grande do Sul and Santa Catarina states (Maia et al. 2015), it is the first record to São Paulo state.

Fuscoporia senex (Nees \& Mont.) GhobadNejhad, Mycotaxon 101: 208. 2007.

Description: Nuñes \& Ryvarden (2000).

Material examined: São Luiz do Paraitinga, Parque Estadual da Serra do Mar, Núcleo Santa Virgínia, Trilha do Poço do Pito, 26.IV.2014, R.M. Pires et al.314 (SP).
Fuscoporia wahlbergii (Fr.) T. Wagner \& M. Fisch., Mycological Research 105(7): 780. 2001. Description: Dai (2010) and Ryvarden (2004).

Materials examined: São Luiz do Paraitinga, Parque Estadual da Serra do Mar, Núcleo Santa Virgínia, Trilha do Ipiranga, 18.IV.2013, R.M. Pires et al. 36 (SP); Trilha do Garcês, 12.II.2014, R.M. Pires et al. 259 (SP).

Inonotus linteus (Berk. \& M.A. Curtis) Teixeira, Revista Brasileira de Botânica 15(2): 126. 1992.

Figs. 2k-m; 3e

Description: Tian et al. (2013).

Material examined: São Luiz do Paraitinga, Parque Estadual da Serra do Mar, Núcleo Santa Virgínia, Trilha do Poço do Pito, 30.X.2013, R.M. Pires et al. 157 (SP).

Remarks: I. linteus is a species complex characterized by pileate basidiomata, a heterogeneous hyphal system with a monomitic context and a dimitic hymenophoral trama, hymenial setae and basidiospores broadly ellipsoid to subglobose, yellowish and thick-walled (Tian et al. 2013). This collection has rare hymenial setae, pore size 5-6 per mm, basidiospores 4.8-6.2 $\times 4.0-4.9 \mu \mathrm{m}$. The basidiospores in our material are bigger than the Tian et al. (2013) descriptions $[4.8-5.5(-5.7) \times(3.8-) 3.9-4.6(-4.8) \mu \mathrm{m}]$, but the name was accepted, since it can be a normal variation within the species. For more accurate resolution, molecular data are needed. It is the first report to São Paulo State, but it is well-known in northeast Brazil (Alagoas, Bahia, Ceará, Paraíba, Pernambuco, Piauí and Sergipe states), and southern Brazil (Paraná and Santa Catarina states) (Maia et al. 2015).

Inonotus portoricensis (Overh.) Baltazar \& Gibertoni, Mycotaxon 111: 206. 2010.

Description: Borba-Silva et al. (2013). Figs. 2a-c; $3 \mathrm{f}$

Material examined: São Luiz do Paraitinga, Parque Estadual da Serra do Mar, Núcleo Santa Virgínia, Trilha do Ipiranga, 17.IV.2013, R.M. Pires et al. 23 (SP).

Additional material examined: BRASIL. PERNAMBUCO: Recife, Parque Estadual de Dois Irmãos, Trilha atrás do casarão, J.M. Baltazar et al., 500 (URM). ALAGOAS: Pilar, RPPN-Fazenda S. Pedro, T.B. Gibertoni, s.n. (URM).

Remarks: The species is characterized by its sessile pilei, applanate to effused-reflexed basidioma with a more or less persistent velvety brown tomentum separated from the context by a distinct dark line (Ryvarden 2004). It presents, besides, setal hyphae $(70-240 \times 8-15 \mu \mathrm{m})$ and basidiospores broadly ellipsoid to ellipsoid and slightly thickwalled $\left(4.8-5.5 \times 3.6-4.2 \mu \mathrm{m}\right.$ and $\left.\mathrm{Q}_{\mathrm{m}}=1.3\right)$, at first 
pale yellow, becoming pale rusty brown with age (Larsen \& Cobb-Poulle 1990). It has been reported to tropical and sub-tropical America; in Brazil, it is known in Alagoas, Bahia, Pernambuco, Rio Grande do Sul and Santa Catarina States (Borba-Silva et al. 2013; Campos-Santana et al. 2015; Maia et al. 2015). This collection represents the first record to São Paulo State.

Inonotus tropicalis (M.J. Larsen \& Lombard) T. Wagner \& M. Fisch., Mycologia 94(6): 1009. 2002. Description: Lowe (1966) and Ryvarden (2004). Materials examined: São Luiz do Paraitinga, Parque Estadual da Serra do Mar, Núcleo Santa Virgínia, Trilha do Ipiranga, 12.VI.2013, R.M. Pires et al. 73 (SP); Trilha do Pirapitinga, 31.X.2013, R.M. Pires et al. 176 (SP).

Additional material examined: BRASIL. RIO GRANDE DO SUL: São Leopoldo, 1904, Rick 14 (S F15659 Holotype), Rick 423 (S F15660 Paratype).

Phellinus grenadensis (Murrill) Ryvarden, Norwegian Journal of Botany 19: 234. 1972.

Figs. 2n-p; $3 \mathrm{~g}$

Description: Ryvarden (2004).

Material examined: São Luiz do Paraitinga, Parque Estadual da Serra do Mar, Núcleo Santa Virgínia, Trilha do Pirapitinga, 11.II.2014, R.M. Pires et al. 240 (SP).

Remarks: It is the first citation for São Paulo state, but known in other seven Brazilian states (Alagoas, Bahia, Pernambuco, Rio Grande do Norte, Rio de Janeiro, Paraná and Santa Catarina) (Maia et al. 2015). P. grenadensis presents perenial and pileate basidiomata, at first reddish brown, tomentose, then becoming crustose, dark brown to dull (Larsen \& Cobb-Poulle 1990). Tubes distinctly stratified separated by context layers. Dimitic hyphal system and hymenial setae absent. Basidiospores ellipsoid, golden yellow to rusty brown $(4.0-6.0 \times 3.0-5.0 \mu \mathrm{m})$.

Phellinus roseocinereus (Murrill) D.A. Reid, Memoirs of the New York Botanical Garden 28(1): 194. 1976.

Figs. 2h-j; $3 \mathrm{~h}$

Description: Ryvarden (2004).

Material examined: São Luiz do Paraitinga, Parque Estadual da Serra do Mar, Núcleo Santa Virgínia, Trilha do Garcês, 12.II.2014, R.M. Pires et al. 258 (SP).

Remarks: this species presents a pileate, sessile, woody, convex to ungulate basidiomata, yellow to reddish brown pore surface (6-10 per $\mathrm{mm}$ ) and basidiospores broadly oval and hyaline (3.5-4.0 × 2.0-3.0 $\mu \mathrm{m})$. Larsen \& Cobb-Poulle (1990) described the presence of hymenial setae 20-30 $\times 4.0-5.0 \mu \mathrm{m}$, in the present collection the setae are smaller $(15-20 \times 4.0-7.0 \mu \mathrm{m})$. Once reported to Pernambuco State, now is cited to São Paulo State (Maia et al. 2015).

Phellinus undulatus (Murrill) Ryvarden, Norwegian Journal of Botany 19: 235. 1972.

Figs. 2d-g; $3 \mathrm{i}$

Description: Lowe (1966) and Loguercio-Leite \& Wright (1995).

Material examined: São Luiz do Paraitinga, Parque Estadual da Serra do Mar, Núcleo Santa Virgínia, Trilha do Pau de Bala, 25.IV.2014, R.M. Pires et al.283 (SP).

Remarks: The species is known from tropical and sub-tropical areas (Ryvarden 2004), and was reported to Alagoas, Pará and Santa Caratina Brazilian states (Maia et al. 2015). This collection is the first citation for São Paulo state. It is characterized by its resupinate basidioma, goldenbrown margin and greyish brown pore surface. Microscopically, the hooked hymenial setae (19-25 $\times 8-12 \mu \mathrm{m})$ are diagnostic, although some straight setae may occur. The basidiospores are broadly ellipsoid and hyaline $(4.1-4.6 \times 3.2-3.6 \mu \mathrm{m})$.

Phylloporia pectinata (Klotzsch) Ryvarden, Synopsis Fungorum 5: 196. 1991.

Description: Ryvarden (2004).

Material examined: São Luiz do Paraitinga, Parque Estadual da Serra do Mar, Núcleo Santa Virgínia, Trilha do Pirapitinga, 8.VIII.2013, R.M. Pires et al. 121 (SP).

\section{Acknowledgments}

The authors are grateful to Fundação de Apoio e Amparo à Pesquisa do Estado de São Paulo, for the scholarship granted to the first author (FAPESP 2012/25493-7). And we are grateful to all Herbaria cited in the text, for the loan of the materials.

\section{References}

Baltazar, J.M. \& Gibertoni, T.B. 2009. A checklist of the aphyllophoroid fungi (Basdiomycota) recorded from the Atlantic Rain Forest. Mycotaxon 109: 439-442.

Borba-Silva, M.A.; Ferreira-Lopes, V. \& DrechslerSantos, E.R. 2013. New records of Flaviporus hydrophilus and Phellinus portoricensis (Fungi: Polypores). Check List 9: 815-817.

Campos-Santana, M.D.; Robledo, G.; Decock, C. \& Silveira, R.M.B.D. 2015. Diversity of the poroid Hymenochaetaceae (Basidiomycota) from the Atlantic Forest and Pampa in Southern Brazil. Cryptogamie, Mycologie 36: 43-78.

Coelho G. 2005. Brazilian new species of Auriporia. Mycologia 97: 266-270. 
da Silva, J.M.C. \& Casteleti, C.H.M. 2003. Status of the biodiversity of the Atlantic Forest of Brazil. In: Galindo-Leal, C. \& Câmara, I.G. (eds.). The Atlantic Forest of South America: biodiversity status, threats, and outlook. CABS and Island Press, Washington. Pp. 43-59.

Dai, Y.C. 2010. Hymenochaetaceae (Basidiomycota) in China. Fungal Diversity 45: 131-343.

Dai, Y.C.; Cui, B.K. \& Decock, C. 2008. A new species of Fomitiporia (Hymenochaetaceae, Basidiomycota) from China based on morphological and molecular characters. Mycological Research 112: 375-380.

Drechsler-Santos, E.R.; Groposo, C. \& Loguercio-Leite, C. 2008. New records of lignocellulolytic fungi (Basidiomycetes) from the Atlantic Rain Forest in the state of Santa Catarina, Brazil. Hoehnea 35: 57-61.

Fidalgo, O. \& Bononi, V.L.R. (coords). 1984. Técnicas de coleta, preservação e herborização de material botânico. Manual 4. Instituto de Botânica, São Paulo. 62p.

Fidalgo, O. 1968. As microestruturas e sua importância na sistemática dos fungos superiores. Rickia 3: 117-159.

Gerber, A.L. \& Loguercio-Leite, C. 1997. New records of Polypores (Aphyllophorales) from Southern Brazil. Mycotaxon 62: 305-318.

Maia, L.C.; De Carvalho Júnior, A.A.; Cavalcanti, L.D.H.; Gugliotta, A. de M.; Drechsler-santos, E.R.; Santiago, A.L.M. de A.; Giachini, A.J.; Soares, A.M. da S.; Gomes-Silva, A.C.; Magnago, A.C.; Goto, B.T.; De Lira, C.R.S.; Salvador-Montoya, C.A.; Pires-Zottarelli, C.L.A.; Da Silva, D.K.A.; Soares, D.J.; Rezende, D.H.C.; Luz, E.D.M.N.; Gumboski, E.L.; Wartchow, F.; Karstedt, F.; Freire, F.M.; Coutinho, F.P.; De Melo, G.S.N.; Sotão, H.M.P.; Baseia, I.G.; Pereira, J.; de Oliveira, J.J.S.; Souza, J.F.; Bezerra, J.L.; Araujo Neta, L.S.; Pfenning, L.H.; Gusmão, L.F.P.; Neves, M.A.; Capelari, M.; Jaeger, M.C.W.; Pulgarín, M.P.; Menolli Jr.; N., De Medeiros, P.S.; Friedrich, R.C.S.; Chikowski, R. dos S.; Pires, R.M.; Melo, R.F.; Silveira, R.M.B. da; Urrea-Valencia, S.; Cortez, V.G. \& da Silva, V.F. 2015. Diversity of brazilian fungi. Rodriguésia 66: 1033-1045. DOI:10.1590/2175-7860201566407.

Gilbertson, R.L. \& Ryvarden, L. 1986. North American Polypores. Oslo, Fungiflora. v.1, p.1-433.

Gilbertson, R.L. \& Ryvarden, L. 1986. North American Polypores. Vol. 1. Fungiflora, Oslo. Pp. 1-433.

Gilbertson, R.L. \& Ryvarden, L. 1987. North American polypores. Vol. 2. Megasporoporia - Wrightoporia. Fungiflora, Oslo. Pp. 1-885.

Góes-Neto, A.; Loguercio-Leite, C. \& Guerrero, R.T. 2000. Taxonomy and qualitative ecological aspects of poroid Hymenochaetales in a Brazilian seasonal tropical forest. Mycotaxon 76: 197-211.

Groposo, C. \& Loguercio-Leite, C. 2005. Contribution to the lignocellulolytic fungi (Basidiomycetes) of the
Atlantic Rain Forest in Southern Brazil. Mycotaxon 92: 103-106.

Groposo, C.; Loguercio-Leite, C. \& Góes-Neto, A. 2007. Fuscoporia (Basidiomycota, Hymenochaetales) in Southern Brazil. Mycotaxon 101: 55-63.

He, S.H. \& Dai, Y.C. 2012. Taxonomy and phylogeny of Hymenochaete and allied genera of Hymenochaetaceae (Basidiomycota) in China. Fungal Diversity 56: 77-93.

Joly, C.A.; Assis, M.A.; Bernacci, L.C.; Tamashiro, J.Y.; Campos, M.C.R. de; Gomes, J.A.M.A.; Lacerda, M.S.; Santos, F.A.M. dos; Pedroni, F.; Pereira, L.S.; Padgurschi, M.C.G.; Prata, E.M.B.; Ramos, E.; Torres, R.B.; Rochelle, A.; Martins, F.R.; Alves, L.F.; Vieira, S.A.; Martinelli, L.A.; Camargo, P.B. de; Aidar, M.P.M.; Eisenlohr, P.V.; Simões, E.; Villani, J.P.; \& Belinello, R. 2012. Florística e fitossociologia em parcelas permanentes da Mata Atlântica do sudeste do Brasil ao longo de um gradiente altitudinal. Biota Neotropica 12: 125-145.

Kirk, P.; Cannon, P.F.; Minter, D.W. \& Stalpers, J.A. 2008. Ainsworth \& Bisby's Dictionary of the Fungi. $10^{\text {th }}$ ed. CAB International, Wallingford. Pp. 1-771.

Larsen, M.J. \& Cobb-Poulle, L.A. 1990. The genus Phellinus (Hymenochaetaceae): a survey of the world taxa. Fungiflora, Oslo. Pp. 1-206.

Loguercio-Leite, C. \& Wright, J.E. 1995. The genus Phellinus (Hymenochaetaceae) on the Island of Santa Catarina, Brazil. Mycotaxon 54: 361-388.

Lowe, J.L. 1966. Polyporaceae of North America. The genus Poria. Technical Publication of the State University College of Forestry at Syracuse University 90: 1-183.

Motato-Vásquez, V. \& Gugliotta, A.M. 2013. Polypores from an Atlantic rainforest area in southeast Brazil: resupinate species. Brazilian Journal of Botany 36 : 211-221.

Motato-Vásquez, V.; Pires, R.M. \& Gugliotta, A.M. 2014. Polypores from an Atlantic rainforest area in southeast Brazil: pileate species. Brazilian Journal of Botany 37: 1-16.

Núñez, M. \& Ryvarden, L. 2000. East Asian polypores. Synopsis Fungorum 13: 1-168.

Ribeiro, M.C.; Metzger, J.P.; Martensen, A.C.; Ponzoni, F.J. \& Hirota, M.M. 2009. The Brazilian Atlantic forest: how much is left, and how is the remaining forest distributed? Implications for conservation. Biological Conservation 142: 1141-1153.

Ryvarden, L. \& Gilbertson, R.L. 1994. European polypores. Part 2. Synopsis Fungorum 7: 394-743.

Ryvarden, L. \& Johansen, I. 1980. A preliminary polypore flora of East Africa. Fungiflora, Oslo. 636p.

Ryvarden, L. 1991. Genera of polypores, nomenclature and taxonomy. Synopsis Fungorum 5: 1-373.

Ryvarden, L. 2004. Neotropical Polypores. Part 1. Introduction, Ganodermataceae \& 
Hymenochaetaceae. Synopsis Fungorum 19. Fungiflora, Oslo. 227p.

Teixeira, A.R. 1962. As microestruturas do basidiocarpo e sistemática do gênero Fomes (Fries) Kickx. Rickia 1: 13-93.

Teixeira, A.R. 1995. Método para estudo das hifas do basidiocarpo de fungos poliporáceos. Manual 6 . Instituto de Botânica, São Paulo. 20p.

Tian, X.M.; Yu, H.Y.; Zhou, L.W.; Decock, C.; Vlasák, J. \& Dai, Y.C. 2013. Phylogeny and taxonomy of the Inonotus linteus complex. Fungal Diversity 58: 159-169.

Valenzuela, R.; Raymundo, T.; Decock, C. \& Esqueda, M. 2012. Aphyllophoroid fungi from Sonora, México 2. New records from Sierra de Álamos-Río Cuchujaqui Biosphere Reserve. Mycotaxon 122: 51-59.

Vlasák, J.; Li, H.J.; Zhou, L.W. \& Dai, Y.C. 2013. A further study on Inonotus linteus complex
(Hymenochaetales, Basidiomycota) in tropical America. Phytotaxa 124: 25-36.

Wagner, T. \& Fischer, M. 2001. Natural groups and a revised system for the European poroid Hymenochaetales (Basidiomycota) supported by nLSU rDNA sequence data. Mycological Research 105: 773-782.

Wagner, T. \& Fischer, M. 2002. Proceedings towards a natural classification of the worldwide taxa Phellinus s. 1. and Inonotus s. 1., and phylogenetic relationships of allied genera. Mycologia 94: 9981016.

Westphalen, M.C. \& Silveira, R.M.B. 2013. Pileate polypores from Araucaria Forests in Southern Brazil. Hoehnea 40: 77-86.

Westphalen, M.C.; Reck, M.A. \& Silveira, R.M.B. 2010. Polypores from Morro Santana, Rio Grande do Sul, Brazil. Hoehnea 37: 647-662. 